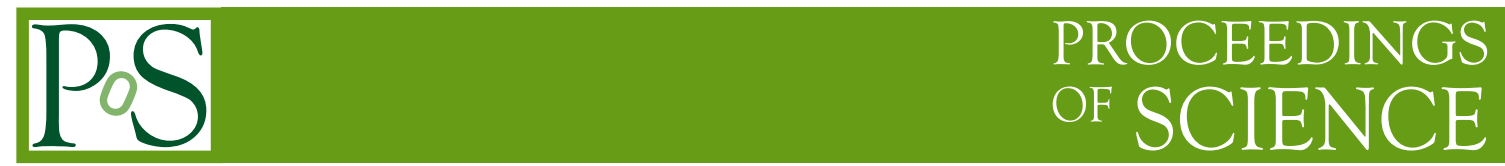

\title{
Hadronic charm meson decays at BESIII
}

\section{Yu-Lan Fan*}

School of Physics and Technology, Wuhan University, Wuhan, P.R.China

E-mail: yulanf@whu.edu.cn

(On behalf of the BESIII Collaboration)

BESIII experiment has collected $e^{+} e^{-}$collision data samples corresponding to integrated lumonisities of $2.93 \mathrm{fb}^{-1}, 3.19 \mathrm{fb}^{-1}$ and $3.13 \mathrm{fb}^{-1}$ at the center-of-mass (c.m.) energies of $3.773 \mathrm{GeV}$, 4.178 GeV, and 4.189-4.226 GeV, respectively. We report the measurements of strong phase differences in $D^{0}$ decays, including $K_{S / L}^{0} \pi^{+} \pi^{-}, K_{S / L}^{0} K^{+} K^{-}, K^{+} \pi^{-} \pi^{-} \pi^{+}$and $K^{-} \pi^{+} \pi^{0}$, which can reduce the systematic uncertainty of $\gamma / \phi_{3}$ measurement at $\mathrm{LHCb}$ and Belle II experiments. In addition, we report the amplitude analyses and measurements of the absolute branching fractions of $D^{0}, D^{+}$and $D_{s}$ decays.

10th International Workshop on Charm Physics (CHARM2020),

31 May - 4 June, 2021

Mexico City, Mexico - Online

${ }^{*}$ Speaker 


\section{Introduction}

BEPCII is a double-ring $e^{+} e^{-}$collider operating at c.m. energy between $2.0 \mathrm{GeV}$ and $4.9 \mathrm{GeV}$, which has reached the design luminosity of $1 \times 10^{33} \mathrm{~cm}^{-2} \mathrm{~s}^{-1}$ in April 2016. BESIII is a major spectrometer running at BEPCII for the studies of hadron physics and $\tau$-charm physics. Charm meson pairs $D \bar{D}\left(D_{s} \bar{D}_{s}^{*}\right)$ are produced near energy threshold $3.773 \mathrm{GeV}$ (4.178 GeV and 4.189$4.226 \mathrm{GeV}$ ) without accompanying particles, corresponding to integrated luminosity of $2.93 \mathrm{fb}^{-1}$ $\left(3.19 \mathrm{fb}^{-1}\right.$ and $\left.3.13 \mathrm{fb}^{-1}\right)$ [1]. Based on these data samples, hadronic $D$ decays can be studies with low background. A double tag (DT) technique is used in most analyses expect 4.4, in which a single tag (ST) technique is used. DT is a method of fully reconstructing $D \bar{D}$ pair, which ST is a method of partially reconstructing a $D$ meson. We report the recent results with precision significantly improved or observation for the first time. Charge-conjugate modes are implied throughout this paper.

\section{Measurements of strong-phase parameters in $D^{0}$ decays}

\section{$2.1 D^{0} \rightarrow K_{S / L}^{0} \pi^{+} \pi^{-}$}

BESIII has reported the determination of strong-phase parameters in $D^{0} \rightarrow K_{S / L}^{0} \pi^{+} \pi^{-}$decay [2,3]. In model-independent GGSZ approach [4], strong-phase parameters measured from quantum-correlated $D^{0} \bar{D}^{0}$ decays are the key input parameters for $\gamma / \phi_{3}$ measurement. Three binning schemes are used in this work, equal $\Delta \delta_{D}\left(\Delta \delta_{D}\right.$ is the relative strong-phase between $D^{0}$ and $\bar{D}^{0} \rightarrow K_{S / L}^{0} \pi^{+} \pi^{-}$), optimal and modified optimal. Two-dimensional fits are performed to extract signal events. In order to enlarge the amount of collected DT events, two partial-reconstruction methods are used by missing one $\pi^{+}$from $D$ and missing one $\pi^{0}$ from $K_{S}^{0}$.

The results of $c_{i}$ and $s_{i}\left(c_{i}\right.$ and $s_{i}$ shown in Fig. 1 are the average $\cos \delta_{D}$ and $\sin \delta_{D}$ in Dalitz plot bin $i$, respectively.) from $D^{0} \rightarrow K_{S / L}^{0} \pi^{+} \pi^{-}$, which are the most precise measurements to date. The strong-phase parameters are still limited by statistical uncertainty. BESIII results are a factor of 1.9 to 2.8 more precise than previous results, and the associated uncertainty on $\gamma / \phi_{3}$ is reduced from 4 degrees to 1 degree. The improved results is important input for $\gamma / \phi_{3}$ measurement in $B$ decay.
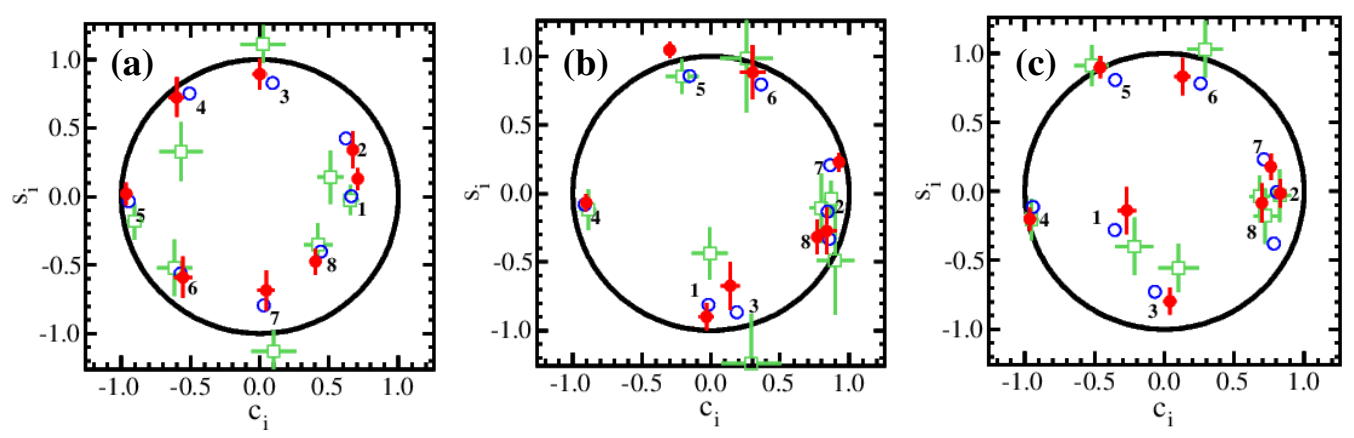

Figure 1: The $c_{i}$ and $s_{i}$ measured from this work, the predictions of Ref.[5] and the results of Ref. [6] corresponding to the red dots with error bars, the black open circles and green squares with error bars, respectively. 
$2.2 D^{0} \rightarrow K_{S / L}^{0} K^{+} K^{-}$

For the strong-phase parameters measured in $D^{0} \rightarrow K_{S / L}^{0} K^{+} K^{-}$decay [7], by using the equal $\Delta \delta_{D}$ binning scheme, the results of strong-phase parameters for $\mathrm{N}=2,3$ and 4 equal $\Delta \delta_{D}$ bins are obtained, which are consistent with the CLEO measurement [6] in all bins and are the most precise measurement to date of strong-phase difference in these decays. For $\mathrm{N}=2,3$ and 4 equal $\Delta \delta_{D}$ binning, the estimated uncertainties caused by the uncertainty of the measured values of $c_{i}$ and $s_{i}$ is $2.3^{\circ}, 1.3^{\circ}$, and $1.3^{\circ}$, respectively. The values are also important for the determination of charm-mixing parameters and search of $\mathrm{CP}$ violation.

2.3 $D^{0} \rightarrow K^{-} \pi^{+} \pi^{+} \pi^{-}$and $D^{0} \rightarrow K^{-} \pi^{+} \pi^{0}$

Recently, BESIII reported a coherence factors and strong-phase differences study of $D \rightarrow$ $K^{ \pm} \pi^{\mp} \pi^{+} \pi^{-}$and $D \rightarrow K^{ \pm} \pi^{\mp} \pi^{0}$ decays [8]. Based on the global analysis and equal $\Delta \delta_{D}$ binning scheme which the phase space is partitioned into 4 pairs of irregularly bin, the coherence factor $(R)$ and average strong-phase difference $(\delta)$ of each decay are measured. The region of parameter spaces $\left(R_{K 3 \pi}, \delta_{D}^{K 3 \pi}\right)$ and $\left(R_{K \pi \pi^{0}}, \delta_{D}^{K \pi \pi^{0}}\right)$ encompassed by 2-3 $\sigma$ confidence intervals are significantly more constrained than the measurements of CLEO-c [9]. The phase space is re-performed in four bins of the $D \rightarrow K^{ \pm} \pi^{\mp} \pi^{+} \pi^{-}$to yield results, to which the $\gamma / \phi_{3}$ is determined with the precision to be around $6^{\circ}$.

\section{Amplitude analyses}

\section{$3.1 D_{s}^{+} \rightarrow \pi^{+} \pi^{0} \eta$}

BESIII has reported the first amplitude analysis of $D_{s}^{+} \rightarrow \pi \pi^{0} \eta$ decay [10] which is a Wannihilation (WA) dominated channel. We retain a sample of $1239 D_{s}^{+} \rightarrow \pi^{+} \pi^{0} \eta$ candidates that has a purity of $(97.7 \pm 0.5) \%$. The WA dominant decays $D_{s}^{+} \rightarrow a_{0}(980)^{+} \pi^{0}$ and $D_{s}^{+} \rightarrow$ $a_{0}(980)^{0} \pi^{+}$are observed for the first time. The measured absolute branching fraction (BF) $\mathcal{B}\left(D_{s}^{+} \rightarrow\right.$ $\left.a_{0}(980)^{+(0)} \pi^{0(+)}, a_{0}(980)^{+(0)} \rightarrow \pi^{+(0)} \eta\right)=\left(1.46 \pm 0.15_{\text {stat. }} \pm 0.23_{\text {syst. }}\right)$ is larger than the BFs of other measured pure WA decays by at least one order of magnitude. Furthermore, the BF of $D_{s}^{+} \rightarrow \pi^{+} \pi^{0} \eta$ is measured with significantly improved precision.

3.2 $D_{(s)}^{+} \rightarrow K_{s}^{0} \pi^{+}\left(K^{+}\right) \pi^{0}$

Using a data sample of 692 DT candidate events with a purity of $97.4 \%$, we perform the first Dalitz plot (DP) analysis of the singly Cabibbo-suppressed (SCS) decay $D^{+} \rightarrow K_{S}^{0} K^{+} \pi^{0}$ [11]. The $\mathrm{DP}$ analysis shows that the $\mathrm{BF}$ of dominated component $K^{*}(892)^{+} K_{S}^{0}$ is $\left(8.69 \pm 0.46_{\text {stat. }} \pm 0.68_{\text {syst. }} \pm\right.$ $\left.0.18_{\text {exet. }}\right) \times 10^{-3}$, which is consistent with the previous results [12] but with a precision improved by a factor of 4.6 .

We perform an amplitude analysis of the $D_{s}^{+} \rightarrow K_{S}^{0} \pi^{+} \pi^{0}$ decay [13] to better understand the vector-pesudoscalar channels of the SCS $D_{s}^{+}$decay. We obtained 609 DT events with a purity of 83.1\%. Amplitudes, fit fractions (FFs) and phases contributing to this final state are measured. The BF for the decay $D_{s}^{+} \rightarrow K_{S}^{0} \pi^{+} \pi^{0}$ is measured to be $\left(5.43 \pm 0.30_{\text {stat. }} \pm 0.15_{\text {syst. }}\right) \times 10^{-3}$ with an improved precision by a factor of 3 compared to the previous measurement [14]. The decay BFs 
with intermediate states like $K^{*}(892)^{0(+)} \pi^{+(0)}$ and $K_{S}^{0} \rho$ are consistent with the theoretical predictions in Ref. [13].

\section{3 $D_{S}^{+} \rightarrow K^{+} K^{-} \pi^{+}\left(\pi^{0}\right)$}

BESIII perform an amplitude analysis of $D_{s}^{+} \rightarrow K^{+} K^{-} \pi^{+}$[16], this results provides important inputs for theory and refines theoretical models. Meanwhile, there are obvious difference between FFs of BaBar and CLEO results [17, 18]. We obtain 4399 DT events with a purity of $99.6 \%$ which means a background free. And a model-independent partial wave analysis in the low $K^{+} K^{-}$ mass region is performed to determine the $K^{+} K^{-}$S-wave lineshape. The BF of $D_{s}^{+} \rightarrow K^{+} K^{-} \pi^{+}$ is determined to be $\left(54.7 \pm 0.08_{\text {stat. }} \pm 0.13_{\text {syst. }}\right) \%$ which is the most precise measurement up to know. The decay BFs with intermediate states like $\bar{K}^{*}(892)^{0} K^{+}$and $\phi(1020) \pi^{+}$are consistent with theoretical predictions [19].

An amplitude analysis of decay $D_{s}^{+} \rightarrow K^{+} K^{-} \pi^{+} \pi^{0}$, which BF has a large systematic uncertainty, has performed by BESIII [20]. This analysis allows to probe the substructures involving $K_{1}(1270)$, $K_{1}(1400)$ and $f_{1}(1420)$ mesons. Using a data sample of 3088 DT events with a purity of $97.5 \%$, the magnitudes, FFs and phases of different components have been determined. For the BFs measurements, the BF of $D_{s}^{+} \rightarrow K^{+} K^{-} \pi^{+} \pi^{0}$ is measured to be $\left(5.42 \pm 0.10_{\text {stat. }} \pm 0.17_{\text {syst. }}\right) \%$ with the precision significantly improved. The BFs of intermediate processes like $\left(\phi \rho^{+}\right)$and $\bar{K}^{* 0} K^{*+}$ are obtained with a much better precision than previous measurements [21,22]. The inconsistence exists between different experiments of the ratio of $K_{1}(1270)$ decay, $R_{K_{1}(1270)}=\frac{\mathcal{B}\left(K_{1}(1270) \rightarrow K^{*} \pi\right)}{\mathcal{B}\left(K_{1}(1270) \rightarrow K \rho\right)}$ [23]. This ratio is measured to be $\left(0.51 \pm 0.12_{\text {stat. }} \pm 0.09_{\text {syst. }}\right) \%$ in this analysis, which is consistent with the results using CLEO's and Belle' data within uncertainties.

\section{Absolute branching fractions}

\section{1 $D \rightarrow \eta X$}

BESIII report the first measurements of the absolute BFs of 14 hadronic decays to exclusive final states with an $\eta$ [24] which are key potential backgrounds in some lepton flavor universality (LFU) tests. The known exclusive $D^{0(+)} \rightarrow \eta X$ decays only account for $44 \%$ (16\%) of their corresponding inclusive rates. Moreover, these decays are crucial to address the tensions found in LFU tests with semi-leptonic $B$ decays, as well as searches for $\mathrm{CP}$ violation. In this analysis, two decay channels with largest BFs are $\mathcal{B}\left(D^{0} \rightarrow K^{-} \pi^{+} \eta\right)=\left(1.853 \pm 0.025_{\text {stat. }} \pm 0.031_{\text {syst. }}\right) \%$ and $\mathcal{B}\left(D^{+} \rightarrow K_{S}^{0} \pi^{+} \eta\right)=$ $\left(1.309 \pm 0.037_{\text {stat. }} \pm 0.031_{\text {syst. }}\right) \%$. The charge-parity asymmetries for the six decays with highest event yields are determined, and so no evidence of $\mathrm{CP}$ violation is found.

\section{2 $D \rightarrow \omega \pi \pi$}

We measure the BFs of SCS decays $D \rightarrow \omega \pi \pi$ [25]. In this analysis, the BF of $D^{0} \rightarrow \omega \pi^{+} \pi^{-}$ is determined to be $\left(1.33 \pm 0.16_{\text {stat. }} \pm 0.12_{\text {syst. }}\right) \times 10^{-3}$ with the statistical significance of $12.9 \sigma$, and corresponding precision highly improved than CLEO measurement. We also measure the BF of $D^{+} \rightarrow \omega \pi^{+} \pi^{0}$, which is $\left(3.87 \pm 0.83_{\text {stat. }} \pm 0.25_{\text {syst. }}\right) \times 10^{-3}$, for the first time with the statistical significance of 7.7 $\sigma$. In addition, no significant signal of $D^{0} \rightarrow \omega \pi^{0} \pi^{0}$ is observed, and the upper limit on the $\mathrm{BF}$ is set to be $\mathcal{B}\left(D^{0} \rightarrow \omega \pi^{0} \pi^{0}\right)<1.10 \times 10^{-3}$ at $90 \%$ confidence level. 


\section{3 $D \rightarrow K \bar{K} \pi \pi$}

We report the first direct measurements of the absolute BFs of nine $D^{0(+} \rightarrow K \bar{K} \pi \pi$ decays containing $K_{S}^{0}$ or $\pi^{0}$ mesons. The $D^{0} \rightarrow K^{+} K^{-} \pi^{0} \pi^{0}, D^{0} \rightarrow K_{S}^{0} K^{-} \pi^{+} \pi^{0}, D^{0} \rightarrow K_{S}^{0} K^{+} \pi^{-} \pi^{0}$, $D^{+} \rightarrow K_{S}^{0} K^{+} \pi^{0} \pi^{0}$ and $D^{+} \rightarrow K_{S}^{0} K_{S}^{0} \pi^{+} \pi^{0}$ decays are observed for the first time, while the BFs of the $D^{0} \rightarrow K_{S}^{0} K_{S}^{0} \pi^{+} \pi^{-}, D^{+} \rightarrow K^{+} K^{-} \pi^{+} \pi^{0}, D^{+} \rightarrow K_{S}^{0} K^{-} \pi^{+} \pi^{+}$and $D^{+} \rightarrow K_{S}^{0} K^{+} \pi^{+} \pi^{-}$decays are measured with improved precision in comparison with the world-average values. Our results can be use to explore $D \bar{D}$ mixing or CP violation and to understand quark $\mathrm{SU}(3)$-flavor symmetry.

\section{4 $D_{s}^{+} \rightarrow P P$}

The BFs $D_{s}^{+}$to two peseudo-scalar mesons $\left(K^{+} \eta^{\prime}, \pi^{+} \eta^{\prime}, K^{+} \eta, p i^{+} \eta, K^{+} K_{S}^{0}, \pi^{+} K_{S}^{0}\right.$ and $\left.K^{+} \pi^{0}\right)$ are measured by analyzing data collected at $\sqrt{s}=4.178-4.226 \mathrm{GeV}$ with BESIII [27], which can be used to explore SU(3) asymmetries and provide crucial calibrations to different theoretical models. The signal yields are extracted by fitting the invariant mass of $D_{s}^{+}$with ST events and the normalization mode $D_{s}^{+} \rightarrow K^{+} K^{-} \pi^{+}$is used in this work. Precision of our measurements are significantly improved in comparison with current world average values.

\section{5 $D^{+} \rightarrow \eta \eta \pi^{+}$and $D^{0(+)} \rightarrow \eta \pi^{+} \pi^{-(0)}$}

We measure the absolute BFs of $D^{+} \rightarrow \eta \eta \pi^{+}, D^{+} \rightarrow \eta \pi^{+} \pi^{0}$ and $D^{0} \rightarrow \eta \pi^{+} \pi^{-}$to be $(2.96 \pm$ $\left.0.24_{\text {stat. }} \pm 0.10_{\text {syst. }}\right) \times 10^{-3},\left(2.23 \pm 0.15_{\text {stat. }} \pm 0.10_{\text {syst. }}\right) \times 10^{-3}$, and $\left(1.20 \pm 0.07_{\text {stat. }} \pm 0.04_{\text {syst. }}\right) \times 10^{-3}$, respectively. The BF of $D^{+} \rightarrow \eta \eta \pi^{+}$is measured for the first time. The BFs of $D^{0(+)} \rightarrow \eta \pi^{+} \pi^{-(0)}$ are consistent with the CLEO's results with improved precision. We also test CP asymmetries of the BFs of $D$ and $\bar{D}$ decays, but no evidence of CP violation is found [28].

\section{Summary}

We report the measurements of the strong-phase parameters in $D^{0}$ decays with best precision, which can reduce the systematic uncertainty for $\gamma / \phi_{3}$ measurements at LHCb and Belle II. In excess of five amplitude analyses of $D$ decays are performed. In addition, more than $20 D$ meson decays are reported for the first time and exceeding $D$ decays are measured with best precision. These results have been used to check $\mathrm{SU}(3)$ asymmetry and to support isospin symmetry, but no CP violation is found. At the c.m. energy $\sqrt{s}=3.773 \mathrm{GeV}$, BESIII plans to take another $17 \mathrm{fb}^{-1}$ data [26]. More results in hadronic charm meson decays can be expected.

\section{References}

[1] M. Ablikim et al. (BESIII Collaboration), Chin. Phys. C 44 (2020), 040001.

[2] M. Ablikim et al. (BESIII Collaboration), Phys. Rev. Lett. 124 (2020), 241802.

[3] M. Ablikim et al. (BESIII Collaboration), Phys. Rev. D 101 (2020), 112002

[4] A. Giri, Y. Grossman, A. Soffer and J. Zupan, Phys. Rev. D 68 (2003), 054018.

[5] I. Adachi et al. (BaBar and Belle Collaboration), Phys. Rev. D 98 (2018), 112012. 
[6] J. Libby et al. (CLEO Collaboration), Phys. Rev. D 82 (2010), 112006.

[7] M. Ablikim et al. (BESIII Collaboration), Phys. Rev. D 102 (2020), 052008.

[8] M. Ablikim et al. (BESIII Collaboration), JHEP 05 (2021), 164.

[9] T. Evans, S. Harnew, J. Libby, S. Malde, J. Rademacker and G. Wilkinson,Phys. Lett. B 757 (2016), 520; Phys. Lett. B 765 (2017), 402 (erratum).

[10] M. Ablikim et al. (BESIII Collaboration), Phys. Rev. Lett. 123 (2019), 112001.

[11] M. Ablikim et al. (BESIII Collaboration), arXiv:2104.09131 [hep-ex].

[12] P. L. Frabetti et al. (E678 Collaboration), Phys. Lett. B 346 (1995), 199.

[13] M. Ablikim et al. (BESIII Collaboration), arXiv:2103.15098 [hep-ex].

[14] . P. Naik et al. (CLEO Collaboration), Phys. Rev. D 80 (2009), 112004.

[15] Y. Fu-Sheng, X. X. Wang and C. D. Lu, Phys. Rev. D 84 (2011), 074019.

[16] M. Ablikim et al. (BESIII Collaboration), arXiv:2011.08041 [hep-ex].

[17] P. del Amo Sanchez et al. (BaBar Collaboration), Phys. Rev. D 83 (2011), 052001.

[18] R. E. Mitchell et al. (CLEO Collaboration), Phys. Rev. D 79 (2009), 072008.

[19] P. del Amo Sanchez et al. (BaBar Collaboration), Phys. Rev. D 83 (2011), 052001.

[20] M. Ablikim et al. (BESIII Collaboration), arXiv:2103,02482 [hep-ex].

[21] P. Avery et al. (CLEO Collaboration), Phys. Rev. Lett. 68 (1992), 1279.

[22] H. Albrecht et al. (ARGUS Collaboration), Z. Phys. C 53 (1992), 361.

[23] M. Artuso et al. (CLEO Collaboration), Phys. Rev. D 85 (2012), 122002; M. Ablikim et al. (BESIII Collaboration), Phys. Rev. D 95 (2017), 072010; R. Aaij et al. (LHCb Collaboration), Eur. Phys. J. C 78 (2018), 443; H. Guler et al. (Belle Collaboration), Phys. Rev. D 83 (2011), 032005; C. Daum et al. (ACCMOR Collaboration), Nucl. Phys. B 187 (1981), 1; P. dArgent et al. JHEP 05 (2017), 143.

[24] M. Ablikim et al. (BESIII Collaboration), Phys. Rev. Lett. 124 (2020), 241803.

[25] M. Ablikim et al. (BESIII Collaboration), Phys. Rev. D 102 (2020), 052003

[26] M. Ablikim et al. (BESIII Collaboration), Chinese Phys. C 44 (2020), 040001.

[27] M. Ablikim et al. (BESIII Collaboration), JHEP 08 (2020), 146.

[28] M. Ablikim et al. (BESIII Collaboration), Phys. Rev. D 101 (2020), 052009. 